FSU-HEP-940311

KEK-TH-392

$\mathrm{MAD} / \mathrm{PH} / 825$

UH-511-785-94

\title{
SUPERCOLLIDER SIGNATURES OF SUPERGRAVITY MODELS WITH YUKAWA UNIFICATION
}

\author{
Howard Baer ${ }^{1}$, Manuel Drees ${ }^{2}$, Chung Kao ${ }^{1}$, \\ Mihoko Nojiri ${ }^{3}$ and Xerxes Tata ${ }^{4}$ \\ ${ }^{1}$ Department of Physics, Florida State University, Tallahassee, FL 32306 USA \\ ${ }^{2}$ Department of Physics, University of Wisconsin, Madison, WI 53706 USA \\ ${ }^{3}$ Theory Group, KEK, Oho 1-1, Tsukuba,Ibaraki 305, Japan \\ ${ }^{4}$ Department of Physics and Astronomy, University of Hawaii, Honolulu, HI 96822 USA
}

(October 18, 2018)

\begin{abstract}
We study the predictions of the simplest SU(5) grand unified model within the framework of minimal supergravity, including constraints from the radiative breaking of electroweak symmetry. As a consequence of the unification of the $b$-quark and $\tau$-lepton Yukawa couplings, the top quark mass is predicted to be close to its fixed point value. We delineate the regions of the supergravity parameter space allowed by constraints from the non-observation of proton decay and from the requirement that the LSP does not overclose the universe. These constraints lead to a definite pattern of sparticle masses: the feature unique to Yukawa unified models is that some of the third generation squarks are much lighter than those of the first two generations. Despite the fact that all sparticle masses and mixings are determined by just four SUSY parameters at the GUT scale (in addition to $m_{t}$ ), we find that the signals for sparticle production can vary substantially over the allowed parameter space. We identify six representative scenarios and study the signals from sparticle production at the LHC. We find that by studying the signal in various channels, these scenarios may be distinguished from one another, and also from usually studied "minimal models" where squarks and sleptons are taken to be degenerate. In particular, our studies allow us to infer that some third generation squarks are lighter than other squarks - a feature that could provide the first direct evidence of supergravity grand unification.
\end{abstract}




\section{INTRODUCTION}

Supersymmetry (SUSY) [1] is increasingly being regarded as the most compelling of the various extensions of the Standard Model proposed to date. SUSY relates properties of bosons and fermions resulting in a new level of synthesis. Supersymmetry also provides a technically natural framework for incorporating the Higgs mechanism crucial for the breaking of electroweak symmetry. On the experimental front, while the measurements of the gauge couplings by the experiments at LEP are inconsistent with minimal models of unification of gauge interactions, these measurements are in good agreement with the predictions of the simplest supersymmetric grand unified model [2]. As an added bonus, supersymmetry provides a natural candidate for cosmological dark matter [3].

Despite these many attractive attributes of supersymmetric theories, no compelling model has as yet emerged. This could be due to the fact that the dynamics of supersymmetry breaking is not yet understood. In phenomenological analyses, supersymmetry breaking is incorporated by the introduction of soft SUSY breaking terms which do not jeopardize the stability of the electroweak mass scale in the presence of radiative corrections from very massive degrees of freedom not included in the SM (these could be the particles of a grand unified theory (GUT), flavour physics at intermediate scales or effects of quantum gravity at the Planck scale). Even in the simplest low energy effective theories, the incorporation of all possible such soft breaking terms [4] consistent with the known low energy symmetries leads to a proliferation of new parameters, greatly limiting the utility of this approach for SUSY phenomenology.

This situation can be greatly ameliorated if the symmetries of the interactions that lead to the breaking of supersymmetry can be guessed at. For example, in a SUSY GUT, if SUSY breaking is assumed to occur above the scale of GUT breaking, the soft SUSY breaking masses and couplings of all particles in the complete multiplet are related. Supergravity (SUGRA) models [5] provide a very attractive realization of this idea of utilizing symmetries to restrict the supersymmetry breaking interactions. It is assumed that SUSY breaking in the effective low energy theory relevant for phenomenology arises due to gravitational interactions, which being universal, allow for only a few independent soft-supersymmetry breaking parameters (a common scalar mass, a gaugino mass along with SUSY breaking trilinear, and possibly, bilinear SUSY breaking scalar coupling) despite our ignorance of the details of the dynamics of SUSY breaking. All this, of course, holds at the ultra-high scale of SUSY breaking. In order to use the structure of the couplings thus obtained for perturbative calculations at the $100 \mathrm{GeV}$ energy scale relevant to experiment today, we must sum the large logarithms arising from the disparity of these scales. This is most conveniently done using the renormalization group technique [6] whereby the soft breaking masses and couplings at the scale of electroweak breaking [7,8] may be obtained. When mixing effects are negligible (as is the case for the first two generations of sfermions) or absent (as for gluinos), these masses only differ slightly from the physical (pole) masses of the sparticles [9]. The real beauty of this framework is that these same radiative corrections drive the desired breakdown of electroweak symmetry, leaving colour and electromagnetic gauge invariance intact. As a bonus, supergravity models lead to (approximate) degeneracies of the first two generations of squarks which is required [10 by the non-observation of flavour changing neutral currents in the $\mathrm{K}$ meson sector. It is interesting that the masses of $t$ - and $b$ - squarks 
may be significantly different; this can lead to interesting phenomenological consequences as we will see below.

While most phenomenological analyses of supersymmetry embody some of the ideas of supergravity GUTs, the observation that the LEP data are consistent with the predictions of SUSY SU(5) has led several groups [7, 8] to systematically study the implications of the simplest SUGRA SU(5) model with radiative symmetry breaking constraints incorporated. It has been pointed out [11] that these same data vitiate one of the earliest successes of non-SUSY SU(5) GUT - the prediction of the ratio $m_{b} / m_{\tau} \simeq 3$ from $b$ - $\tau$ Yukawa coupling unification. In contrast, the data are in agreement with the Yukawa coupling unification in the SUGRA SU(5) framework provided that the top quark mass is close to its infrared fixed point [12 14.

The purpose of this paper is to study the implications of the simplest SU(5) supergravity model with radiative symmetry breaking constraints incorporated. We will mainly focus on the detectability of the sparticle signals at the Large Hadron Collider (LHC) and especially, on whether these signals serve to distinguish this framework from the usually studied "minimal SUSY" framework [15,[16]. Here, by simplest, we mean that we assume the fewest number of particles and the smallest number of soft-SUSY breaking parameters at the scale $M_{X}$ of GUT symmetry breaking. More specifically, we assume:

1. A minimal set of soft SUSY breaking parameters at the GUT scale $M_{X} \simeq 2 \cdot 10^{16} \mathrm{GeV}$, i.e. a common scalar mass $m_{0}$, a common gaugino mass $m_{1 / 2}$ and common trilinear $\left(A_{0}\right)$ and bilinear $\left(B_{0}\right)$ soft breaking parameters. We do not assume any relation between $A_{0}$ and $B_{0}$, though.

2. Minimal particle content at and below the scale $M_{X}$.

We recognize that these assumptions are sensitive to the unknown physics at high energy scales, and may well prove to be incorrect; for instance, the assumption of a common gaugino mass might be invalid if there are non-minimal kinetic terms for the gauge fields [17]. Our point, however, is that at present even this very restrictive model can be claimed to be realistic in the sense it is compatible with all experimental constraints both from particle physics as well as from cosmology. Moreover, as we will see, the fact that the top quark Yukawa couplings are driven to their fixed point values results in supercollider signals that are characteristically different from previous studies [16]. Despite the fact that our analysis entails an extrapolation of physics over a very large range of energy, we believe that the simplicity of the model and the characteristic predictions of the scenario provide sufficient motivation for our analysis.

In the next section, we discuss how the two assumptions together with various observational constraints from collider and non-accelerator experiments as well as from cosmological considerations further constrain the model parameters in this already restrictive framework. We also recapitulate how the sparticle spectrum and the SUSY parameters of the low energy theory are obtained and present representative examples of these. In section III, we study the general features of the various scenarios obtained with our assumptions, and discuss how these differ from those usually considered in phenomenological analyses within the Minimal Supersymmetric Standard Model (MSSM) framework. In the next section we briefly describe our simulation of sparticle production at hadron colliders using ISAJET 7.07 18] and discuss various improvements that we have recently incorporated into the program. In 
Section V, we discuss various SUSY signals that might be expected at the Large Hadron Collider (LHC) in the representative scenarios introduced above. We show that an observable signal may be expected in various event topologies including in single lepton and dilepton $+\#_{T}$ samples, which are usually thought to be obscured by large SM backgrounds. Here, and in the next section, we discuss how the simultaneous observation of a signal in several channels can serve to differentiate the SUGRA SU(5) from the MSSM framework, and also, to distinguish between the various illustrative supergravity scenarios. We conclude with a summary of our results and some general remarks.

\section{THE SUPERGRAVITY SU(5) FRAMEWORK: DESCRIPTION OF CONSTRAINTS}

In this section, we first overview the relations between mass parameters at the weak scale in the minimal SUGRA model. We then describe various theoretical and experimental constraints that we impose, and show that despite these, it is possible to find viable solutions even in our theoretically restricted scenario.

At the high scale $M_{X}$, we start [7],8] with the common gaugino mass $m_{1 / 2}$, the common scalar mass $m_{0}$, the higgsino-mixing parameter $\mu_{0}$, the soft SUSY breaking trilinear (bilinear) couplings $A_{0}\left(B_{0}\right)$, together with the Yukawa couplings $h_{t}, h_{b}$ and $h_{\tau}$ (with $h_{b}=h_{\tau}$ at scale $M_{X}$; see below). The absence of any intermediate scales implies that all parameters at the weak scale can be determined uniquely from the input parameters at $M_{X}$, by solving a set of renormalization group equations (RGE) [6]. Of course, the above set of parameters should reproduce the correct size of symmetry breaking, or $M_{Z}, m_{t}$ (as a input), $m_{b}$ and $m_{\tau}$ at the weak scale; this leads to constraints between the parameters at $M_{X}$. We do not assume any relationship between $A_{0}$ and $B_{0}$. In practice we use the ratio of vacuum expectation values $\tan \beta$ as an input parameter. Using the equations describing the minimization of the Higgs potential at the weak scale then allows us to express $B$ and $|\mu|$ at the weak scale in terms of $m_{Z}$ and $\tan \beta$ [7]; note that the renormalization group equations for $B$ and $\mu$ decouple from all other RGE. The constraint of proper electroweak symmetry breaking therefore reduces the number of free input parameters to five, which we take to be $m_{0}, m_{1 / 2}, A_{0}, h_{t}$ and $\tan \beta$; in addition, the sign of $\mu$ needs to be specified.

These considerations lead to simple relations between gaugino mass parameters $\left(M_{i}\right)$ and first and second generation sfermion masses at the weak scale. The RG running of the masses of strongly interacting particles implies large positive corrections to squark and gluino mass parameters, while corrections to the electroweak interacting particles stay relatively small. Numerically, $m_{\tilde{q}}^{2} \simeq m_{0}^{2}+6 m_{1 / 2}^{2}$, and the soft SUSY-breaking gaugino masses are related by $M_{1} \simeq 0.4 m_{1 / 2}, M_{2} \simeq 0.8 m_{1 / 2}$ and $M_{3} \simeq 2.7 m_{1 / 2}$.

The Higgs mass parameters and third generation squark masses receive significant radiative corrections from Yukawa interactions in addition to those from gauge interactions. At $M_{X}$ our ansatz requires the two Higgs mass parameters $m_{H_{1}}$ and $m_{H_{2}}$ to be equal:

$m_{H_{i}}^{2}=m_{0}^{2}+\mu_{0}^{2}$. As is well known, this equality implies that the tree-level potential cannot simultaneously lead to nonzero vacuum expectation values and be bounded from below. Quantum effects, i.e. the running of the mass parameters of the scalar potential, thus become crucial for gauge symmetry breaking. Radiative $S U(2) \times U(1)$ symmetry breaking is in fact a natural prediction of this model, given the large size of the top Yukawa coupling. 
More explicitly, $m_{\mathrm{H}_{2}}^{2}$ receives a negative radiative correction proportional to $h_{t}^{2}$ times some function of soft SUSY breaking parameters when going towards the weak scale, due to its interaction with the top quark. On the other hand $m_{H_{1}}^{2}$ is insensitive to $h_{t}$, thereby lifting the equality between Higgs mass parameters. If $h_{t}$ is very large, $m_{H_{2}}^{2}$ becomes negative well above the weak scale, resulting in too large a value of $m_{Z}$ or even an unbounded Higgs potential. This can be compensated by choosing $|\mu|$ to be large. In other words, $|\mu|$ has to be increased along with $h_{t}$ in order to maintain $M_{Z}$ at its correct value.

We now turn to the constraints coming from our assumption of a minimal SUSY SU(5) GUT. The minimality of the $S U(5)$ Higgs sector immediately implies the equality of the bottom and tau Yukawa couplings at the unification scale. Several recent studies [12 [14] have shown that this is only compatible with the measured values of $b$ quark and $\tau$ lepton masses if the top Yukawa coupling is large, i.e. close to its "fixed point" value [19]. This conclusion persists even if the ratio $h_{b} / h_{\tau}$ is varied about unity by $10 \%$ to account for uncertainties due to threshold effects. This immediately leads to the prediction

$$
m_{t}\left(m_{t}\right) \simeq 190 \mathrm{GeV} \cdot \sin \beta
$$

where $m_{t}\left(m_{t}\right)$ is the running $(\overline{\mathrm{MS}})$ mass. Since we only use 1-loop RGE everywhere, we simply interpreted eq.(2.1) to mean

$$
h_{t}\left(M_{X}\right)=2 .
$$

Taking the on-shell top quark mass $m_{t} \leq 190 \mathrm{GeV}$ [20], we find $\tan \beta \leq 2.5$ [12], in general agreement with the constraints on it from nucleon decay [21] which we now turn to.

In SUSY GUTs, the exchange of higgsino triplets can lead to a dangerously short lifetime of the proton. In minimal SUSY $S U(5)$ its exact value depends on many parameters. In particular, the lifetime increases with increasing mass of the higgsino triplet. Since the dimension -5 operators induced by higgsino triplet exchange need to be "dressed" by loops involving squarks and gauginos (chargino loops usually being dominant), contributions from (s)quarks of different generations in the loop add coherently. This opens the possibility to increase the total lifetime, at the cost of some fine-tuning, by cancelling contributions involving third generation (s)quarks against those involving only the first two generations. The "dressing loop function" can be decreased, and hence proton decay can be suppressed, by choosing scalars (squarks and sleptons) to be considerably heavier than gauginos. For given values of the other parameters the lower bound on the proton lifetime therefore translates into an upper bound on the ratio $m_{1 / 2} / m_{0}^{2}$. In view of the various uncertainties [22] of this calculation we have conservatively required [21],

$$
m_{0} \geq \min \left(300 \mathrm{GeV}, 3 m_{1 / 2}\right) .
$$

The coefficient 3 on the right hand side has been chosen so that the contribution $m_{0}^{2}$ to $m_{\tilde{q}}^{2}$ described earlier dominates the one $\propto m_{1 / 2}^{2}$, which implies $m_{\tilde{q}} \gg m_{1 / 2}$.

Finally, we require our model to be cosmologically acceptable. Because the MSSM has a conserved R parity, the lightest supersymmetric particle (LSP) is stable. Thus the LSP can be a good candidate for the missing cosmological mass density, or Dark Matter (DM). Large $|\mu|$ means that the lightest neutralino, which is also LSP in this scenario, is dominantly a gaugino. The constraint eq.(2.3) implies that LSP annihilation via the $t$-channel exchange 
of sfermions is suppressed, leading to a dangerously large relic abundance (or small age of the Universe) unless LSP annihilation is enhanced by the proximity of an $s$-channel pole. This is not as unnatural as it sounds, since recent studies [23] have shown very large pole enhancements even if the LSP mass is several GeV below half the mass of the boson exchanged in the $s$-channel. Our large values of $\mu$ and $m_{0}$ and the rather small value of $\tan \beta$ force most Higgs bosons to be heavy, the exception, of course, being the lighter neutral scalar $H_{\ell}$. This is because $m_{H_{1}}^{2}$ does not receive large negative radiative correction from Yukawa interactions if $\tan \beta$ is small, while $m_{H_{p}}^{2}=m_{H_{1}}^{2}+m_{H_{2}}^{2}$ with $m_{H_{2}}^{2}>-m_{Z}^{2} / 2$ from the minimization of the Higgs potential. The condition eq.(2.3) then implies $m_{H_{p}}>10 m_{\mathrm{LSP}}$. Therefore only the $Z$ boson and the light Higgs boson can contribute significantly to LSP annihilation.

In our computation of the Higgs sector, we have included one loop corrections from top and bottom (the latter are small) Yukawa interactions to both masses and trilinear Higgs interactions using the effective potential [24]; note that these corrections are not contained in the RGE discussed above. Despite the large top Yukawa coupling, we find that $m_{H_{\ell}}$ is bounded from above by about $110 \mathrm{GeV}$ for the values of $\tan \beta$ we are considering. Moreover, $H_{\ell}$ is always SM-like in this scenario, due to the large masses of the other Higgs bosons. Hence the LEP bound of $62.5 \mathrm{GeV}$ applies for it as well. This then leads to bounds on the mass of a cosmologically acceptable LSP, and hence on the gaugino mass:

$$
60 \mathrm{GeV} \leq\left|m_{1 / 2}\right| \leq 130 \mathrm{GeV} .
$$

Finally, we impose the limit

$$
m_{0} \leq 1 \mathrm{TeV}
$$

in order to avoid excessive fine tuning.

We should mention a technical complication that arises from the constraint (2.3) as a result of which squarks are substantially heavier than gluinos. It has been shown [9] that the pole gluino mass receives additional corrections proportional to $\log \left(m_{\tilde{q}} / m_{\tilde{g}}\right)$. Since one of the features of these scenarios, as we will see, is a rather light gluino, these extra corrections - which can be as large as 30\% - can be significant. We have incorporated these into our program using the formulae in Ref. [9].

Our apparently innocuous assumption of minimal particle content at the GUT scale led to the various constraints on model parameters as we have just discussed. Most analyses require either the equality of $b$ and $\tau$ Yukawa couplings at $M_{X}$, or an acceptably long lifetime for the proton, but not both. It seems to us that these two constraints are really on very similar theoretical footing in that they can both be weakened or even avoided completely if one allowed a more complicated Higgs sector and/or a larger GUT group [25]. On the other hand, we do not try to find correlations between the value of the strong coupling constant and the overall size of SUSY breaking masses. Given the present (mostly theoretical) error of $\alpha_{s}\left(M_{Z}\right)$, and in view of further uncertainties at the high scale we do not consider any "bounds" on sparticle masses derived in this fashion to be reliable at present. We now turn to a discussion of the characteristics of the fixed point scenario outlined above, and give some illustrations of the spectra and the sparticle mixing patterns that may be anticipated in this class of models. 


\section{THE SUPERGRAVITY SU(5) FRAMEWORK: SPARTICLE MASSES AND MIXING PATTERNS}

The characteristic feature of the fixed point scenario of the last section is the large top Yukawa coupling, eq. (2.1). This, as we have seen, leads to $|\mu| \geq m_{0}$, assuming the radiative breaking mechanism of electroweak symmetry. Together with eq. (2.3), this then implies that the lighter (heavier) charginos and neutralinos are gaugino- (higgsino-) like in character. The limit eq. (2.4) implies that gluinos cannot be much heavier than about $400 \mathrm{GeV}$ and may even be light enough to be discovered at the Tevatron. We have also seen that the pseudoscalar $\left(H_{p}\right)$, the charged $\left(H^{ \pm}\right)$and the heavier neutral scalar $\left(H_{h}\right)$ Higgs bosons all tend to be heavy, so that these will not be produced via cascade decays of gluinos [26]. Finally, we make the obvious remark, $m_{\tilde{q}}>m_{\tilde{g}}$ so that the squarks of the first two families dominantly decay to gluinos.

Another important consequence of the large top family Yukawa interactions is the sizable reduction of the SUSY-breaking masses of scalar top quarks, and further, the mass squared of $\tilde{t_{R}}$ is reduced twice as much as that of $\tilde{t_{L}}$. By $S U(2)$ invariance this means that the mass of left-handed scalar bottom quarks will also be reduced compared to squarks of the first two generations. The top squark masses are further altered by squark mixing, which depresses the lighter state even further. This non-degeneracy of third generation squarks, which is one of the characteristic features of minimal SUGRA models with radiative gauge symmetry breaking is accentuated by the large value of $h_{t}$ in the model.

In spite of the rather strong constraints on parameter space, we found that there is still considerable room for variation in the expected phenomenology. While condition (2.3) implies that masses of the first and second generation squarks are well above the gluino mass no such statement can be made for the lighter stop eigenstate $\tilde{t_{1}}$. In particular, it is possible that the two body decay $\tilde{g} \rightarrow t+\tilde{t_{1}}$ is allowed [27], in which case it will have a branching ratio near $100 \%$, being the only two-body decay mode of $\tilde{g}$. We remind the reader that it has been shown in Ref. [28] that signals from a relatively light $t$-squark may well have escaped detection at the Fermilab Tevatron, although with the accumulation of a large data sample, its detection may be possible [29].

Despite the various constraints that we have imposed it is impossible to do an exhaustive scan of parameter space. Moreover, for the purposes of this study, the main question is not whether it is possible to detect supersymmetry at the LHC (for the light spectrum we have this has already been answered affirmatively [16]), but whether it is possible to distinguish the present class of models from the usually studied MSSM, and further, whether it is possible to distinguish the various scenarios (discussed shortly) from one another. Toward this end, we have attempted to choose model parameters (respecting the constraints discussed in Sec. II) that accentuate the qualitative differences in the signals.

At hadron colliders, squarks and gluinos are the most copiously produced particles. Since, within our framework, the first two generations of squarks dominantly decay to gluinos, we expect that the gluino decay patterns fix the broad features of the phenomenology. This immediately leads us to consider two classes of scenarios: $(\mathrm{A})$ where the decay $\tilde{g} \rightarrow t+\tilde{t_{1}}$ is allowed, and (B) where it is forbidden. Case A naturally subdivides into two cases determined by the decay pattern of the lighter $t$-squark. In case $\mathrm{A} 1$, we assume that the decay $\tilde{t}_{1} \rightarrow b \widetilde{W}_{1}$ is accessible; since this is the only tree-level two body decay, its branching 
fraction is essentially $100 \%$. In case A2, we assume that the decay $\tilde{t}_{1} \rightarrow b \widetilde{W}_{1}$ is kinematically inaccessible; in this case the stop decays [30] via one loop diagrams into a charm jet and an LSP: $\tilde{t}_{1} \rightarrow c \widetilde{Z}_{1}$.

Turning to the heavier stop, case B, we have attempted to diversify the phenomenology by choosing combinations of $m_{0}$ and $m_{1 / 2}$ at their maximum and minimum values discussed in Sec. II. This leads to four cases B1-B4.

- B1: $m_{1 / 2}$ and $m_{0}$ small,

- B2: $m_{1 / 2}$ small; $m_{0}$ large,

- B3: $m_{1 / 2}$ large; $m_{0}$ small,

- B4: $m_{1 / 2}$ and $m_{0}$ large.

Notice that for the case B1 where both squarks and gluinos are at their minimum phenomenologically acceptable values, the gluino may even be accessible at the Tevatron. Our point in analysing this case is more to illustrate the diversity of phenomena that could result even in this restricted framework, and to study the range of parameters over which distinguishable signals may be possible at the LHC.

The input parameters, the resulting spectra and the values of $\mu$ at the weak scale in these six cases are shown in Table I. Several features are worth pointing out.

- We see that the gluinos are relatively light. As noted above, gluino signals in case B1 (and, possibly, B2) may even be observable at the Tevatron.

- It is striking to see that in case $\mathrm{A} 2, m_{\tilde{t}_{1}}$ is only $84 \mathrm{GeV}$; a significant number of $\tilde{t}_{1}$-pair events should, therefore, be present in the existing data sample at the Tevatron. As pointed out in Ref. [28] such a light stop squark may well have escaped detection at the Tevatron especially since the LSP is rather heavy in this case. It should, however, be remembered that both the experiments at the Tevatron have since accumulated at least $15-20 p b^{-1}$, so that the detectability of the stop at the Tevatron merits a reevaluation 229]. It would indeed be interesting to study whether stop squark signals in cases A and B2 might be detectable at the Tevatron.

- The lighter chargino and the second lightest neutralino in B1 and B2 should be kinematically accessible at LEP II. For the other cases in Table I, their accessibility will obviously depend on the energy that LEP II is finally able to attain. Since, as remarked above, both $\widetilde{Z}_{1}$ and $\widetilde{Z}_{2}$ are dominantly gaugino-like (and selectrons are heavy), their production at LEP II will be suppressed. For cases B1 and B2, the trilepton signal from $\widetilde{W}_{1} \widetilde{Z}_{2}$ production at the Tevatron should be present at observable rates. In the other cases illustrated, the trilepton cross section (after experimental cuts) is $\sim 1 \mathrm{fb}$ [31], and hence, is unlikely to be observable. This is because we have $m_{\tilde{\ell}} \simeq m_{\tilde{q}}$ so that, unlike as in no-scale models, the leptonic branching ratios are not dynamically enhanced.

- The light Higgs boson $H_{\ell}$ is essentially the SM Higgs boson. It should, therefore, certainly be detectable at LEP II in case B1, while the detectability in the other cases will be sensitive to the machine energy that is ultimately achieved. 
- We note that the top quark is necessarily within the reach of the Tevatron, and further, its decays are as given by the SM except in case A2 where the decay $t \rightarrow \tilde{t}_{1} \widetilde{Z}_{1}$ has a branching fraction of $<10 \%$.

- Finally, we see that as noted above, $m_{\tilde{b}_{L}}$ is indeed considerably smaller than the mass of the first two generations of squarks. This implies that three body gluino decays mediated by $\tilde{b}_{L}$ exchange are enhanced relative to those mediated by other squarks. As a result, gluino events in cases $\mathrm{B}$ may be expected to have a higher multiplicity of $B$ mesons than would be expected if all squarks are taken to be degenerate as in most MSSM studies. It is amusing to note that $\tilde{g} \tilde{g}$ events may be even richer in $B$ 's for cases A, since then, decays of $t$ quarks from $\tilde{g} \rightarrow \tilde{t}_{1} t$ as well as the decays $\tilde{t}_{1} \rightarrow b \widetilde{W}_{1}$ (in case A1) lead to $B$ meson production. We also note that because $\tilde{b}_{L}$ is relatively light, $\widetilde{Z}_{2} \rightarrow b \bar{b} \widetilde{Z}_{1}$ decays can also be considerably enhanced relative to $\widetilde{Z}_{2} \rightarrow d \bar{d} \widetilde{Z}_{1}$ decays. We thus see that the presence of light third generation squarks leads to SUSY events that should be rich in $B$ mesons. Such events may also occur within the MSSM framework where cascade decays of gluinos to Higgs bosons can result in events with a high multiplicity of $b$ quarks [32. However, these cascade decays can occur only when $m_{\widetilde{Z}_{2}}-m_{\widetilde{Z}_{1}}$ exceeds the lighter Higgs boson mass which, for the SM Higgs bosons, is bounded below by $63.5 \mathrm{GeV}$ [33]. Thus, within the MSSM framework, these events occur only when gluinos are rather heavy. In other words, the detection of $B$-rich gluino events with event kinematics corresponding to gluino masses smaller than 400 $\mathrm{GeV}$ could signal a light third generation of squarks.

Next, we briefly describe the improvements that we have made in ISAJET 7.07 18 to simulate supersymmetry events at hadron colliders that we use to compute event rates, topologies and kinematic distributions for the six cases listed in Table I.

\section{SIMULATION OF SUPERSYMMETRY AT HADRON COLLIDERS}

The main production processes and decay modes of all sparticles as given by the minimal supersymmetric model have been incorporated into ISAJET [18 which we use for all our simulations. In order to allow for the possibility that the third generation of squarks may be substantially lighter than the first two generations, which is the distinguishing characteristic of the scenario, we have had to make several modifications to the code. In ISAJET 7.07,

1. we have included the production and decays of $t$-squarks.

2. We have modified the input parameters to the program. In addition to the SM parameters (including $m_{t}$ ) the current low energy inputs in terms of which sparticle masses and decay patterns are computed are $\left(m_{\tilde{g}}, m_{\tilde{q}}, m_{\tilde{\ell}_{L}}, m_{\tilde{\ell}_{R}}, m_{\tilde{\nu}}, \tan \beta, \mu, m_{H_{p}}, m_{\tilde{t}_{L}}\right.$, $\left.m_{\tilde{t}_{R}}, A_{t}, m_{\tilde{b}_{R}}, A_{b}\right)$. The last five entries are new modifications and allow for independent mass and mixing pattern for the third squark generation. The stop and sbottom masses are the respective soft SUSY-breaking mass parameters and not the physical squark masses. Since $\tilde{b}_{L}$ and $\tilde{t}_{L}$ belong to the same $\mathrm{SU}(2)$ doublet, $m_{\tilde{b}_{L}}=m_{\tilde{t}_{L}}$. We have neglected D-term contributions that break the degeneracy between the L- and Rtype squarks of the first two generations but have retained these in the computation of 
slepton masses - this is the only reason for the difference in the $\tilde{\ell}_{L}$ and $\tilde{\nu}_{\ell_{L}}$ masses. For heavy squarks, this D-term splitting is generally small, and so, makes little difference in the computations of squark production cross sections or gluino, chargino and neutralino decays mediated by virtual squarks. This splitting can be very important when squarks and gluinos are close in mass, since then some of the two-body gluino decay modes may become accessible. In the present study, this is never the case because of the constraint (2.3).

3. The input gluino mass is the physical pole mass [9] as opposed to the running $\overline{M S}$ mass that enters the gaugino-Higgsino mass matrices. In all the cases in table I, the pole mass is somewhat larger than the $\overline{M S}$ mass, primarily because the squarks are heavier than gluinos. For Case B2, where the gluino is light and squarks are at their maximal value, this difference is almost $50 \mathrm{GeV}$.

4. In the computation of the Higgs boson masses and couplings, we have, as already mentioned, incorporated radiative corrections from both top and bottom Yukawa interactions, including mixing between third generation squarks. We have not included effects of virtual gauge boson and gaugino loops, but these are known to be small [34].

5. We have supplemented the various sparticle decay formulae to account for nondegenerate third generation sparticles. This can be especially important for gluino three body decays, as well as the corresponding neutralino decays. We have also included contributions to three body $\widetilde{Z}_{2}$ decays mediated by Higgs bosons; these can be important for large values of $\mu$ and $m_{\tilde{q}}$ for which the contributions from both $Z$ and squark mediated decays are dynamically suppressed. As a result, $\widetilde{Z}_{2}$ decays to heavy quarks and leptons may be enhanced.

For every set of input parameters, the routine ISASUSY calculates all sparticle masses along with branching fractions for all their allowed decay modes. Of course, the input parameters are correlated by the minimal SU(5) supergravity constraints discussed above. We then use ISAJET to generate supersymmetric particle pairs in $p p$ collisions. In each run, ISAJET is set up to simultaneously generate all allowed sparticle pairs in numbers proportional to the corresponding pair production cross section. We use the EHLQ Set I structure functions 350 for our computations of cross sections at the LHC. The produced sparticles are then decayed via various cascades with branching fractions as obtained from ISASUSY into final states involving quarks, leptons, gluons and photons. Radiation off initial and final state partons as well as heavy flavour fragmentation is included in ISAJET. Daughter quarks and gluons are hadronized, and unstable particles decayed into the final state. Finally, underlying soft scattering and hadronization of beam remnants is modelled in our simulation.

For each of the six cases in Table I, we use ISAJET to generate a sample of 50K SUSY events by $p p$ collisions at $\sqrt{s}=14 \mathrm{TeV}$. The relative contributions of the different SUSY processes to this event sample is shown in Table II. We see that for the light stop cases A1 and A2, the production of stops dominates, and furthermore, that the stops have a significant production rate even in most of the cases B. The production of other flavours of squarks is suppressed relative to that of gluinos primarily because of Eq.(2.3). We have also shown the branching fractions for the main decay modes of sparticles that are most 
abundantly produced (either directly or via cascades) at the LHC. In cases A1 and A2, the gluino essentially always decays to $t$-squarks whereas in cases $\mathrm{B}, \widetilde{W}_{1}$ and $\widetilde{Z}_{2}$ decays are favoured [36]. We also see that gluino decays to $b$ quarks are enhanced relative to the corresponding decays to $d$ quarks. This is because $\tilde{b}_{L}$ is lighter than the other down type squarks. This enhancement is small for cases B1 and B2 because the $\widetilde{Z}_{1} b \tilde{b}_{L}$ coupling is suppressed by accidental cancellations - these cancellations do not occur for gluino decays

to $\widetilde{Z}_{2}$. Notice also that the decays $\widetilde{Z}_{2} \rightarrow b \bar{b} \widetilde{Z}_{1}$ are also enhanced relative to $\widetilde{Z}_{2} \rightarrow d \bar{d} \widetilde{Z}_{1}$ decays, in part for the same reason, but also because of $H_{\ell}$ exchange diagrams. Finally, we have also shown the branching fraction for the leptonic decays of $\widetilde{W}_{1}$ and $\widetilde{Z}_{2}$ which enter the computation of the multilepton signals. Notice that the neutralino branching fraction exhibits substantial variation while that for the chargino is essentially determined by the $\mathrm{W}$ branching fraction except in Case A2 where the chargino decays via the two body $\tilde{t}_{1} b$ mode.

In order to simulate experimental conditions at the LHC, we impose cuts described in the next section and then classify the signals according to their lepton content as in Ref. [16]. Since the efficiency for SUSY events to pass these cuts depends strongly on the masses and decay patterns of the produced sparticles, the contribution of the various processes after cuts is, in general, quite different from the relative rates in Table II. To compare the features of sparticle production in the supergravity SU(5) model under study, with the corresponding production in "minimal SUSY" models usually [16.15] used to study SUSY signatures, we repeat our simulation for four illustrative cases. Here, we take all squarks and sleptons to be degenerate, and fix the gluino mass at $300 \mathrm{GeV}$, a value similar to $m_{\tilde{g}}$ in the six cases in Table I. Since the signals are known to be sensitive to $m_{\tilde{q}} / m_{\tilde{g}}$, we illustrate our results for $m_{\tilde{q}}=m_{\tilde{g}}+20 \mathrm{GeV}$ and $m_{\tilde{q}}=2 m_{\tilde{g}}$. To vary the electroweak gaugino content we study two values of $\mu$. Specifically, the parameters for these four cases are:

- BTW1: $m_{\tilde{g}}=300 \mathrm{GeV}, m_{\tilde{q}}=320 \mathrm{GeV}, \mu=-150 \mathrm{GeV}$,

- BTW2: $m_{\tilde{g}}=300 \mathrm{GeV}, m_{\tilde{q}}=600 \mathrm{GeV}, \mu=-150 \mathrm{GeV}$,

- BTW3: $m_{\tilde{g}}=300 \mathrm{GeV}, m_{\tilde{q}}=320 \mathrm{GeV}, \mu=-500 \mathrm{GeV}$,

- BTW4: $m_{\tilde{g}}=300 \mathrm{GeV}, m_{\tilde{q}}=600 \mathrm{GeV}, \mu=-500 \mathrm{GeV}$.

For comparison purposes, and in keeping with parameters of Ref. [16], we have fixed $\tan \beta=2$, again a value similar to the cases in Table I, and chosen $m_{t}=140 \mathrm{GeV}, m_{H_{p}}=500$ $\mathrm{GeV}$, with the $A$-parameters set to zero. A discussion of our simulation along with the various results forms the subject of the next section.

\section{SUPERGRAVITY SU(5) SIGNALS AT THE LHC}

As emphasized in Ref. [16], several interesting signals should simultaneously be present if any new physics signal at the LHC is to be attributed to squark or gluino production. These signals include the canonical $\not_{T}$ events, the like-sign dilepton events and multilepton events. These leptonic events should contain substantial jet activity. The production of charginos or neutralinos in association with a squark or gluino yields the same event topologies, while chargino-neutralino production could, in addition, lead to multilepton events free of jet activity. In this section, we study the rates and distributions for these various event 
topologies for the six supergravity cases in Table I and compare these with the "minimal supersymmetry" test cases listed above.

We use the toy calorimeter simulation package ISAPLT to model detector effects. We simulate calorimetry with cell size $\Delta \eta \times \Delta \phi=0.1 \times 0.1$, which extends between $-5<\eta<5$ in pseudorapidity. We take electromagnetic energy resolution to be $10 \% / \sqrt{E_{T}} \oplus 0.01$, while hadronic resolution is $50 \% / \sqrt{E_{T}} \oplus 0.03$ for $|\eta|<3$, and $100 \% / \sqrt{E_{T}} \oplus 0.07$ for $3<|\eta|<5$, where $\oplus$ denotes addition in quadrature. Jets are coalesced within cones of $R=\sqrt{\Delta \eta^{2}+\Delta \phi^{2}}=0.7$ using the ISAJET routine GETJET. Hadronic clusters with $E_{T}>50 \mathrm{GeV}$ are labelled as jets. Muons and electrons are classified as isolated if they have $p_{T}>20 \mathrm{GeV},|\eta(\ell)|<2.5$, and the visible activity within a cone of $R=0.3$ about the lepton direction is less than $E_{T}$ (cone) $=5 \mathrm{GeV}$. Finally, we assume (perhaps optimistically) that it is possible to identify $b$-jets with $p_{T}>20 \mathrm{GeV}$ within $|\eta|<2$ with an efficiency of $40 \%$ via micro-vertex tagging.

Following Ref. [16], we group the various signal categories according to the number of isolated leptons, and further, require $\mathscr{H}_{T}>100 \mathrm{GeV}$ for the SUSY signal. The various signals are,

(i) the $E_{T}$ signal consisting of $0 \ell+n_{j}$ jets $+E_{T}$ events. We require $E_{T}>150 \mathrm{GeV}$, $n_{j} \geq 4$ and the transverse sphericity $S_{T}>0.2$

(ii) the single lepton signal consisting of events with one and only one isolated lepton $\left(1 \ell+j e t s+E_{T}\right)$

(iii) events with two isolated leptons $\left(2 \ell+j e t s+E_{T}\right)$, which can subdivide into opposite sign dileptons (OS) and same sign dileptons (SS).

(iv) events with three isolated leptons $\left(3 \ell+j e t s+\mathbb{E}_{T}\right)$;

$(v)$ and events with four isolated leptons $\left(4 \ell+j e t s+E_{T}\right)$.

Table III gives an overview of the cross sections for these event topologies for the six SUGRA cases in Table I along with the cross sections for the major SM backgrounds. Also shown are the corresponding cross sections for the four "MSSM" cases introduced in Sec. IV. The cross sections shown here include the various cuts mentioned above, as well as additional cuts (discussed below) for the $E_{T}$, the $1 \ell$ and the OS dilepton signals which are known to have large SM backgrounds. As we will see, these additional cuts are essential to suppress the SM background, particularly to the $1 \ell$ and $\ell^{+} \ell^{-}$signals to acceptable levels. We see from Table III that for all but the $1 \ell$ signal in Case B4, the signal to background ratio exceeds unity, and is frequently considerably larger. Furthermore, we see that the cross sections in the $E_{T}, 1 \ell$ and dilepton channels range from $1-100 \mathrm{pb}$ so that $10^{4}-10^{6}$ signal events may be expected annually at the LHC even for a luminosity of $10^{4} \mathrm{pb}^{-1} /$ year. We also see that except for case A2, several thousand trilepton events may be expected every year at the LHC. We have been unable to identify any significant SM physics background to this signal. Finally, we note that while there is some variation of the signals for the different scenarios in Table III, these cross sections alone do not enable us to unambiguously distinguish between all the various cases, or for that matter even between the SUGRA and the "MSSM" cases. This requires a more detailed study of the various signals which we now turn to. 


\section{A. $\not \psi_{T}$ signal}

This is generally regarded as the canonical signal for supersymmetry. Backgrounds in this channel can be very dependent on detector details, especially forward calorimetry, and realistic BG estimates require a detailed calorimeter simulation. Nevertheless, we estimate backgrounds from several major SM sources using our toy calorimeter and ISAJET: $t \bar{t}$ pair production, for $m_{t}=160 \mathrm{GeV}, W+$ jets production, $Z+$ jets production, and heavy flavor $(b \bar{b}$ and $c \bar{c})$ production. Considerable background rejection with relatively little cost to signal can be gained by requiring, in addition,

- $\Sigma\left|E_{T}\right|>700 \mathrm{GeV}$, where the sum is over all calorimeter cells.

The impact of this cut on the signal and the various backgrounds is shown in the $\mathbb{E}_{T}$ column of Table IV. The values (inside) outside the parenthesis indicate the signal cross section (before) after this additional cut. While this cut is not essential in that the signal to background ratio substantially exceeds unity even before the cut, we see that implementing the cut results in a significant improvement of this ratio with only a modest loss of signal. The largest $E_{T}$ cross sections come from the low $m_{1 / 2}$ (light gluino) cases, where the total production cross sections are largest. In order to facilitate distinction between the different scenarios in Table III, we exhibit various characteristics of the $E_{T}$ events in Table V. In columns 3-5, we list the fraction of events with jet multiplicities $n_{j}=4-5, n_{j}=6-7$ and $n_{j} \geq 8$. The most noticeable difference occurs in the $n_{j} \geq 8$ channel, where we see several SUGRA cases yield large fractions of events with very high jet multiplicity. This is especially true of cases A1, A2 and B4. For instance, in A1, many of the events come from gluino pair production, where $\tilde{g} \rightarrow t \tilde{\tilde{t}}_{1} \rightarrow b q q^{\prime}+b q q^{\prime} \widetilde{Z}_{1}$, which can yield up to twelve 'parton level' jets. Of course, it is very difficult for the SM background processes to yield such high jet multiplicities.

We also list in Table $\mathrm{V}$ the fraction of events containing identifiable displaced $b$-vertices, $n_{b}$. An important point is that supersymmetry events often have high multiplicity of $B$ hadrons [16,32] in the central region. This is even more so in the SUGRA cases we have generated. We see that in the SUGRA cases, from $25 \%-65 \%$ of the $\not_{T}$ events have at least one identifiable $B$ vertex, compared to $15 \%-25 \%$ for the BTW cases (of course, larger B multiplicities are also possible in the BTW cases when cascade decays of gluinos into Higgs bosons become operative. However, this occurs for considerably larger values of $m_{\tilde{g}}$.). Also, the SUGRA cases can have large fractions of events with $n_{b} \geq 2$ and $n_{b} \geq 3$. There are several reasons for this:

- in case A1 with the richest $B$ content, each gluino in gluino pair events decays via $\tilde{g} \rightarrow t \tilde{t}_{1}$, which automatically yields at least four $B$ 's per event since the $t$-quark as well as the $\tilde{t}_{1}$ squark decay into a $b$,

- the fact that $\tilde{b}_{L}$ is generally lighter than the first two generation squarks means that three body gluino decay via a virtual $\tilde{b}_{L}$ is enhanced, yielding a large branching fraction for $\tilde{g} \rightarrow b \bar{b} \widetilde{Z}_{i}[8]$,

- neutralino three body decays such as $\widetilde{Z}_{2} \rightarrow b \bar{b} \widetilde{Z}_{1}$ are frequently enhanced, either because of the smaller $\tilde{b}_{L}$ mass, or due to the importance of Higgs boson mediated diagrams discussed in Sec. IV. 
Of course, the exact $b$ multiplicity will depend on the tagging efficiency that is ultimately attained. The ability to $\operatorname{tag} B$ vertices may also be very helpful in obtaining an enriched SUSY sample, and thus aid in SUSY particle mass estimates.

We also show in Table $\mathrm{V}$ the mean summed scalar $E_{T}$ and mean $E_{T}$ in $E_{T}+$ jets events. Use has already been made of $\Sigma\left|E_{T}\right|$ to enhance SUSY signal against background. The scalar $E_{T}$ distribution is somewhat sensitive to the magnitude of the SUSY particle masses produced in the subprocesses although this is not clear from just the mean values listed in Table $\mathrm{V}$. We also remark that the differences between the various cases are somewhat reduced by the hard $\Sigma\left|E_{T}\right|$ cut. We show in Fig. 1 the $p_{T}$ distribution of the fastest jet in $E_{T}$ events, for four SUGRA cases, and the sum of backgrounds. The SUGRA cases have $p_{T}$ which peaks around $\sim 200-250 \mathrm{GeV}$, with a broad tail extending to higher $p_{T}$ 's, unlike SM background. Since squarks of the same mass are expected to lead to harder jets than gluinos, it is natural to ask whether squark production shows up as a shoulder in this distribution. A glance at Fig. 1 shows that this will be difficult. We remark though that the hard cuts that we have imposed to enhance the signal selects out events with similar kinematics and so reduces the differences between the various signals.

\section{B. Single isolated lepton events}

Little effort has gone into searches for SUSY in the single lepton channel, due in part to large backgrounds expected from $W+$ jets production and $t \bar{t}$ production. Within our framework, the production rate of (the rather light) gluinos is truly enormous at the LHC so that one may hope to be able to make rather stringent cuts to eliminate the backgrounds, leaving behind an observable signal. A glance at the cross sections in parenthesis listed in the $1 \ell$ column of Table IV shows that the single lepton cross section is smaller than the SM background except in case B1. To facilitate further separation between the SUSY signal and the SM background, we show in Fig. $2 a)$ the $\Sigma\left|E_{T}\right|$ and $\left.b\right)$ transverse mass $m_{T}\left(\ell, E_{T}\right)$ distributions for four SUGRA cases A1, A2, B1 and B4, along with the summed background distribution. The scalar $E_{T}$ distribution is substantially harder than SM background. Since both the top and the $W$ backgrounds dominantly contain one leptonically decaying $W$ boson, the transverse mass distribution for the background rises to a Jacobian peak at $m_{T}=M_{W}$ while that for the signal is relatively smooth. Hence, to maximize signal against background, we further require:

- $\Sigma\left|E_{T}\right|>700 \mathrm{GeV}$, and

- $m_{T}\left(\ell, E_{T}\right)<60 \mathrm{GeV}$, or $m_{T}\left(\ell, \not_{T}\right)>100 \mathrm{GeV}$.

The resultant cross sections are shown in Tables III and IV, in the $1 \ell$ column. The total SM background is $\sim 12 p b$, while signal ranges between $10-77 p b$. In order to get some idea of the hardness of the leptons in the $1 \ell$ sample, the $p_{T}(\ell)$ distribution after all the above cuts is shown in Fig. 2c. In case B1, where single leptons are apt to come from light chargino decay, a distortion of the low $p_{T}(\ell)$ distribution can occur; for other cases, where leptons come from $t$-quark decay, the distribution more nearly tracks the shape of the background.

Various characteristics of single lepton events are detailed in Table VI. The general features are similar to those of Table V. The jet multiplicity is again large, especially for 
events with larger gluino masses, while jet multiplicities for SM backgrounds are substantially smaller. Furthermore, the SUSY signal events, especially for cases A1, A2 and B4, are characterized by large fractions with visible displaced $B$ vertices. The SUGRA cases, except for case B2, all have $n_{b} \geq 2$ substantially larger than the corresponding BTW values, and hence could yield evidence for a SUGRA-type of sparticle spectrum, where some of the third generation squarks are far lighter than squarks of the first two generations.

\section{Opposite-sign (OS) isolated dilepton events}

As for the case of single lepton events, searches for SUSY in the isolated opposite sign dilepton channel have rarely been considered due to a large expected background from $t \bar{t}$ events. Within our framework, however, we see from the cross sections in parenthesis in the OS column in Table IV that, with just the canonical cuts discussed above, the dilepton signal exceeds that background in the favourable cases A1, B1 and B2. Furthermore, it is only between a factor 2-6 smaller than the background in the other cases. In Fig. $3 a$ we plot the $\Sigma\left|E_{T}\right|$ distribution, for which the signal gives a substantially harder spectrum than background. We see that the additional requirement,

$$
\text { - } \Sigma\left|E_{T}\right|>700 \mathrm{GeV} \text {, }
$$

reduces the background by a factor of seven, with only modest loss of signal, particularly for the cases A2, B3 and B4 where the signal was considerably below background. The cross sections after all cuts are shown in Tables III and IV, in the OS columns. Signal exceeds background for cases A1, B1 and B2, and is comparable in the other SUGRA cases. The $p_{T}(\ell)$ distribution for fast and slow leptons after the above cuts is shown in Fig. $3 b$ and $3 c$. The overall hardness of these distributions depends in part on the SUSY sources of the dileptons. In cases A1 and A2, many of the leptons are quite hard, coming from top quark decay, while in cases B2 and B4 the leptons frequently come from three body chargino and neutralino decay, and exhibit a softer spectrum. For OS dileptons produced in gluino and squark events, either the leptons each separately come from the two SUSY particles produced in the hard scattering, or the two leptons can come from a single SUSY particle cascade, such as $\tilde{g} \rightarrow \widetilde{Z}_{2} \rightarrow \ell \bar{\ell} \widetilde{Z}_{1}$. In the former case, the dilepton pair is expected to have a large opening angle in the transverse plane, whereas in the latter case, the opening angle is expected to be small. This is borne out in the behavior of Fig. 4. In cases B2 and to a smaller extent in case B4 (cases B1 and B3 are similar to B1 and B4, respectively), where there is substantial neutralino production via cascade decays, the dileptons come with a small opening angle. In case A1 the dileptons are somewhat peaked at large opening angle, though the peak is not as pronounced as in cases B2 and B4. This is because the OS dileptons can come from either the same gluino (from the leptonic decay of its $t$ and $\tilde{t}_{1}$ daughters) or from different gluinos and the cuts tend to favour leptons from top decays to those from the decays of stop.

We show OS lepton event characteristics in Table VII. The SUGRA cases again show large jet multiplicity, and large $B$ hadron multiplicity; the latter quantity can again serve to distinguish SUGRA events from the MSSM BTW cases.

Just as with dilepton opening angle, the SUSY source of OS dileptons is correlated with the dilepton flavor asymmetry, defined as 


$$
A_{f}=\frac{N(e \bar{e})+N(\mu \bar{\mu})-N(e \bar{\mu})-N(\bar{e} \mu)}{N(e \bar{e})+N(\mu \bar{\mu})+N(e \bar{\mu})+N(\bar{e} \mu)}
$$

SS dilepton events are expected to have $A_{f} \sim 0$ (replacing $\bar{\ell}$ with $\ell$ ), reflecting uncorrelated sources of the leptons; however, OS dileptons may be expected to have a large positive $A_{f}$ if many of the OS dileptons come from e.g. $\widetilde{Z}_{2} \rightarrow \ell \bar{\ell} \widetilde{Z}_{1}$ decay. The asymmetry is listed in the last column of Table VII. Indeed, cases B1 and B2 have large $A_{f}$, while A1 and A2 have $A_{f}$ consistent with zero. Cases B3 and B4 show intermediate values of $A_{f}$. Notice that the flavour asymmetry qualitatively tracks the azimuthal angle peaking illustrated in Fig. 4.

\section{Same-sign (SS) isolated dilepton events}

The same-sign isolated dilepton signature has been recognized [37,16] for several years now as a particularly clean signal for SUSY, owing to tiny SM backgrounds. It is indicative of the Majorana nature of the gluino; in a gluino pair production event, both gluinos may decay to charginos of the same sign so that subsequent chargino leptonic decay leads to the same-sign dilepton signature. The cross sections after the cuts discussed above are listed in Table III, under the SS column. The SUGRA cases yield cross sections between 0.4-3.7 $p b$ at the LHC, which would yield 4000-37,000 events for an integrated luminosity of $10^{4}$ $p b^{-1}$ per year. Backgrounds are expected to come from $t \bar{t}$ production, where a lepton from $b$ decay is somehow recorded as isolated and from $W^{ \pm} W^{ \pm}$production. Our simulation of $300 \mathrm{~K}$ $t \bar{t}(160)$ events gives a background of $0.01 \mathrm{pb}$, well below signal whereas the corresponding background from same sign $W W$ production has been shown to be [38.16] $\simeq 0.035 p b$. We thus expect that this sample is essentially free of physics backgrounds.

Each lepton in a SS dilepton event usually originates from the cascade decay of different particles produced in the hard scattering subprocess. Thus, the transverse SS dilepton opening angle $\phi\left(\ell \ell^{\prime}\right)$ distribution should be maximal for large opening angles. This is shown by the $\phi$ distribution plotted in Fig. 4. This behavior, in comparison to that of OS dileptons, reflects the source of the dilepton parentage, and hence may be useful in ultimate event reconstruction. Furthermore, if SS and OS dileptons had the same source, for instance as in $\tilde{g} \tilde{g}$ events, then one would expect roughly equal rates for SS and OS dilepton production. In Table III, we present the ratio of OS/SS event rates, where the same cuts have been made for each topology, i.e. the $\Sigma\left|E_{T}\right|$ cut has been removed for OS dileptons. We see in case A2, where dileptons come mainly from $t$ decay in gluino pair events with $\tilde{g} \rightarrow t \tilde{t}_{1}$, that this is indeed the case. In case A1, leptonic decays of the top and stop daughters of the same gluino are an additional source of OS dileptons. In cases B1 and B2, where OS dileptons come frequently come from $\widetilde{Z}_{2} \rightarrow \ell \bar{\ell} \widetilde{Z}_{1}$ decay, the OS/SS ratio is $\sim 6-8$. Cases B3 and B4 yield intermediate values of this ratio, in agreement with Fig. 4 and the flavour asymmetry values in Table VII. Finally, we show in Fig. 5 the fast and slow lepton $p_{T}$ distributions for SS events. The hardness of these distributions again reflects the source of the dileptons. In particular, the slow lepton distribution in OS dilepton events is typically softer than the corresponding distribution in SS events. This is reasonable since there is less energy for multi-leptons produced in the same SUSY particle cascade decay. 


\section{E. Three and Four isolated lepton events}

Complicated superparticle cascade decays can lead to events with 3, 4 and even 5 isolated leptons [16] if gluinos are heavy enough. These cross sections, after the $E_{T}>100 \mathrm{GeV}$ cut, are shown in the $3 \ell$ and $4 \ell$ columns of Table III. We should warn the reader that in our simulation, we have just 10-80 trilepton signal events depending on the case in question and just a handful of $4 \ell$ events. These numbers could, therefore, contain significant statistical fluctuations, and should be viewed in proper perspective. We see that except in case A2, $\sim\left(10^{3}-10^{4}\right)$ trilepton events should be expected in a data sample of $10 \mathrm{fb}^{-1}$ at the LHC. However, since gluinos are relatively light within our framework, the $4 \ell$ cross sections are rather small. SM backgrounds to these multi-lepton topologies are small: for our simulation of $300 \mathrm{~K} t \bar{t}$ events, no isolated multi-leptons were found, leading to an upper bound on BG from this source of $<0.004 \mathrm{pb}$. Estimates of backgrounds from $t \bar{t} t \bar{t}$ and three and four vector boson production also yield rates below the $f b$ level [16].

In the $3 \ell$ channel, we see a wide range of possible rates for our SUGRA cases. Case A2, which is dominated by $\tilde{t}_{1} \tilde{t}_{1}$ and $\tilde{g} \tilde{g}$ production, where $\tilde{g} \rightarrow t \tilde{t}_{1}$ and $\tilde{t}_{1} \rightarrow c \widetilde{Z}_{1}$, produced no $3 \ell$ or $4 \ell$ events, corresponding to an upper limit on this rate of $\sim 0.02 p b$. Case A1, which is similar to A2 except now $\tilde{t}_{1} \rightarrow b \widetilde{W}_{1}$, can yield substantial $3 \ell$ and $4 \ell$ rates in gluino pair events where both tops and stops decay leptonically. Cases B1 and B2, where $\widetilde{Z}_{2} \rightarrow \ell \bar{\ell} \widetilde{Z}_{1}$ is common, can also produce large rates for $3 \ell$ and $4 \ell$ events. Cases B3 and B4 do not produce large rates for $3 \ell$ or $4 \ell$ events due to a small $\widetilde{Z}_{2} \rightarrow \ell \bar{\ell} \widetilde{Z}_{1}$ branching ratio (see Table II).

\section{SUMMARY AND CONCLUDING REMARKS}

In this paper, we have studied the implications of the simplest supergravity grand unified model (based on SU(5) gauge symmetry) with radiative electroweak symmetry breaking for experiments at the LHC. This necessarily involves assumptions about physics at the ultrahigh energy scale. Our assumptions, which include the minimality of the particle content at and below the GUT scale together with the unification of soft-SUSY breaking parameters at the unification scale, are carefully elucidated in Sec. II. In the framework of Minimal Supergravity, the SUSY parameters at low energy can all be obtained by renormalization group evolution of just four GUT scale parameters together with the top quark Yukawa coupling at the unification scale. We have further restricted the range of these parameters by incorporating constraints from the non-observation of proton decay [Eq. (2.3)] and from the requirement that the relic LSP density is compatible with the present age of our universe [Eq. (2.4)]. We have taken at face value the implications of the equality of the $b$-quark and $\tau$-lepton Yukawa couplings at the unification scale (allowing for $10 \%$ deviations from exact equality due to threshold effects) which, as has been pointed out by several groups, implies that the top quark mass is close to its fixed point value.

As discussed in Sec. III, these requirements lead to a distinct pattern of sparticle masses and mixings. We find,

- the gluino is light, $m_{\tilde{g}} \leq 400 \mathrm{GeV}$;

- the lighter $t$-squark is substantially lighter than all other squarks, and further, $\tilde{b}_{L}$ is lighter than the other squarks; 
- the Higgsino mass parameter $|\mu|$ is large, so that the lighter charginos and neutralinos are dominantly gauginos, with masses smaller than about $100 \mathrm{GeV}$;

- $\tan \beta$ is close to unity;

- the lightest scalar in the Higgs sector is never significantly heavier than $100 \mathrm{GeV}$.

We have seen that these mass and mixing patterns lead to characteristic features in SUSY events at hadron supercolliders. Of course, these characteristics can be mimicked by appropriately adjusting the multitude of parameters of the MSSM. The point, however, is that an observation of several of these features could serve as a strong indication of the simplest unification scenario.

Despite the various theoretical and experimental constraints that we have imposed, the parameter space is too large to allow a systematic exploration. Instead, we have identified six sets of model parameters (that respect all the constraints in Sec. II) which, we believe, capture the diversity of signals that may arise within this framework. The input parameters along with the sparticle masses are summarized in Table I. In Cases A1 and A2 the gluino decays via $\tilde{g} \rightarrow t \tilde{t}_{1}$, while in cases $\mathrm{B}$, gluinos decay via three body decays. The main difference between $\mathrm{A} 1$ and $\mathrm{A} 2$ is in the stop decay patterns: in case A1, $\tilde{t}_{1} \rightarrow b \widetilde{W}_{1}$ while in case $\mathrm{A} 2, \tilde{t}_{1} \rightarrow c \widetilde{Z}_{1}$.

Our main results for the signals from the supergravity framework are presented in Sec. V. In order to compare signals from supergravity SU(5) with those of the usually studied "minimal models", we have also studied the same signals for the four scenarios labelled BTW1-BTW4 defined in Sec. IV, which have a similar value of $m_{\tilde{g}}$ as in Table I, but have squarks and sleptons exactly degenerate. As usual, we have classified the signals by their leptonic content. Table III gives an overview of the various signals after all the cuts discussed in Sec. V. We see that there are observable signals in essentially all the channels shown including the $1 \ell$ and $\ell^{+} \ell^{-}$channels which are usually thought to be swamped by SM backgrounds. Unambiguous distinction between all the various supergravity cases, or for that matter, even between SUGRA and BTW cases, is not possible, on the basis of cross sections alone.

In order to facilitate this distinction, we have listed the characteristics of the $E_{T}, 1 \ell$ and $\ell^{+} \ell^{-}$event samples in Tables V, VI and VII, respectively. A major difference between our supergravity cases A1, A2, B3 and B4, and the BTW cases, is that the supergravity events are significantly richer in $B$ hadrons. Of course, whether distinction of scenarios based on $B$ identification is actually possible will crucially depend on the ability of the detectors to identify displaced vertices. In our calculation, we have assumed a $40 \%$ efficiency of identifying central $B$ mesons with $p_{T}>20 \mathrm{GeV}$ in the central region. The reason that supergravity events are $B$-rich is that one, or more, of the third generation of squarks is significantly lighter than the other squarks. In cases A1 and A2 where the gluinos decay via $t \tilde{t}_{1}$, each gluino pair event contains at least two $b$ quarks from the decays of the top (in case A1, the decay of each of the stops also gives $b$ 's). In cases B1-B4, the enrichment of the $B$ sample arises in part because $\tilde{b}_{L}$ is lighter than the other squarks. As a result, gluino and neutralino decays mediated by a virtual $\tilde{b}_{L}$ are enhanced. Furthermore, since $\widetilde{Z}_{1}$ and $\widetilde{Z}_{2}$ are mainly gaugino-like, the Z-mediated decays of $\widetilde{Z}_{2}$ are strongly suppressed. Then, when squarks are very heavy, contributions from Higgs mediated $\widetilde{Z}_{2} \rightarrow b \bar{b} \widetilde{Z}_{1}$ decays, which further 
enrich the $b$ sample, become significant. Finally, in cases B2-B4, B's can also come from $\tilde{g} \rightarrow \widetilde{W}_{1} t b$ decays, although in case B2, the primary $b$ quark is likely to be soft. In cases B3 and B4, the primary $b$ quark and, in all three cases, the $b$ quark from top decay should have large efficiency to pass our cuts. BTW type events can be very rich in $B$ 's if $H_{\ell}$ can be produced via cascade decays. As we have mentioned, this is unimportant for the smaller gluino masses of interest here (presumably, the kinematic features of the events will give a rough measure of gluino mass). We also see from Table $\mathrm{V}$ that in cases A1 and A2, and also in cases B3 and B4, supergravity events can have very high jet multiplicities - about 10\% of the $E_{T}$ sample has $n_{j} \geq 8$ whereas this number is somewhat smaller for the BTW cases. For cases A1 and A2, this is due mainly to the production of top quarks in gluino decays, which leads to a large jet multiplicity. Finally, we see that the flavour asymmetry $A_{f}$ defined in Sec. $\mathrm{V}$ is generally larger in the BTW cases than in the SUGRA cases, except when the gluino is very light. This asymmetry, which is a measure of the rate for neutralino production in gluino and squark cascades, is greatly reduced when chargino cascades dominate as, for instance, in cases A1 and A2.

The features of Tables V-VII also facilitate some distinction between the various supergravity scenarios. Clearly A1 and A2 are readily distinguishable via the high B-multiplicity in the $E_{T}$ sample and also from the absence of any flavour asymmetry in the $\ell^{+} \ell^{-}$sample. The ratio of SS to OS dilepton production rate in Table III may facilitate further distinction. Also, the $B$-multiplicity is significantly higher in A1 than in A2. The dilepton cross section is considerably higher in case A1 (where stops can also yield leptons) than in A2, while exactly the opposite is true for the $E_{T}$ signal. Furthermore, since $\widetilde{Z}_{2}$ rarely occurs in the A1 and A2 cases, case A2 yields no $3 \ell$ or $4 \ell$ cross section, while A1 yields such events only from top and stop decay.

Distinction amongst the various cases B is somewhat more subtle. B1 and B2 are very similar, except for the squark mass, as are B3 and B4. A striking difference between (B1, B2) and $(\mathrm{B} 3, \mathrm{~B} 4)$ is in the flavour asymmetry, in the OS/SS ratio in Table III, in the differential distribution shown in Fig. 4, and especially in the large rate for isolated $3 \ell$ and $4 \ell$ events (as has been explained, these are all measures of $\widetilde{Z}_{2}$ production via cascade decays). Some distinction may also be possible via the scalar $E_{T}$ distribution since the parent gluino masses are significantly different. We should mention that the hard scalar $E_{T}$ cut tends to wash out this difference; therefore, this difference may best be studied in the $E_{T}$ sample prior to this cut. This should be possible since the signal is larger than background even before this cut as can be seen from Table IV. The multiplicity of $B$ mesons may provide yet another distinction. To distinguish B1 from B2 or B3 from B4 is more difficult. This is not surprising since essentially the only difference comes from the squark mass. The clearest distinction appears to be in the cross section for the $1 \ell$ signal and, in the case of B3 and B4, also from the $E_{T}$ cross section. It is encouraging to see that the various scenarios are distinguishable even in the messy environment of a hadron collider.

The reader may have noticed that even though the $t$-squark is light and even though its production constitutes a substantial portion of all SUSY events (see Table II), we have not extracted any direct signal for its production. In fact, signals for $\tilde{t}_{1}$-pair production may even be observable at the Tevatron in several of the cases discussed in this paper. Since our primary purpose here was to study the distinguishability of various scenarios within the SU(5) supergravity framework from one another and from the so-called "minimal 
models", we used rather hard cuts to obtain clean event samples. The direct signals from the lighter sparticles are essentially removed by these cuts and, in our analysis, the existence of the lighter stops and sbottom quarks manifested itself in the decay patterns of the parent gluinos. It may, of course, well be possible to devise a strategy that is dedicated to look for the light scalar top signal at the LHC. Charginos and neutralinos may also be searched for using specialized strategies [39].

In summary, we have examined the phenomenology of the minimal SU(5) supergravity grand unified model with radiative breaking of electroweak symmetry. We have delineated the range of parameters allowed by constraints from proton decay and from a cosmologically acceptable LSP. We have shown that these lead to a characteristic pattern of sparticle masses and mixings. We have examined signals from the production of supersymmetric particles at the LHC for representative points in the parameter space. We find that these signals are distinguishable from the corresponding signals within the usually studied models where squarks and sleptons are taken to be degenerate. We further find that by simultaneously examining various signals it should be possible to distinguish between the various scenarios that are possible even within the restricted framework of supergravity SU(5). Discovery of supersymmetric signals at the LHC with patterns corresponding to any of these cases would be extremely dramatic, since it would not only signal the discovery of supersymmetry, but would provide support for the ideas underlying supergravity grand unification.

Note added: As we were completing this manuscript, we received a paper addressing some of the phenomenological consequences of Yukawa unified "no-scale" models [4].

\section{ACKNOWLEDGMENTS}

We thank M. Bisset for embedding Higgs radiative corrections into ISAJET, and R. Arnowitt, P. Nath and F. Paige for discussions. This research was supported in part by the U. S. Department of Energy under contract numbers DE-FG05-87ER40319, DE-AC0276ER00881 and DE-AM03-76SF00235. The work of HB was supported by the TNRLC SSC Fellowship program. The work of MD was supported in part by the Wisconsin Research Committee with funds granted by the Wisconsin Alumni Research Foundation, by the Texas National Research Laboratory Commission under grant RGFY93-221, as well as by a grant from the Deutsche Forschungsgemeinschaft under the Heisenberg program. XT acknowledges support from the US National Science Foundation's US-Japan Cooperative Research Program. 


\section{REFERENCES}

[1] For phenomenological reviews of supersymmetry, see H. Haber and G. Kane, Phys. Rep. 117, 75 (1985); X. Tata, in The Standard Model and Beyond, p. 304, edited by J. E. Kim, World Scientific (1991); V. Barger and R. J . N. Phillips, Wisconsin preprint, $\mathrm{MAD} / \mathrm{PH} / 765$

[2] P. Langacker and M. Luo, Phys. Rev. D44, 817 (1991); U. Amaldi, W. de Boer and H. Fürstenau, Phys. Lett. B260, 447 (1991); J. Ellis, S Kelley and D. Nanopoulos, Phys. Lett. B260, 131 (1991);

[3] H. Goldberg, Phys. Rev. Lett. 50, 1419 (1983); J. Ellis et. al. Nucl. Phys. B238, 453 (1984).

[4] L. Girardello and M. Grisaru, Nucl. Phys. B194, 65 (1982).

[5] H. P. Nilles, Phys. Rep. 110, 1 (1984); P. Nath, R. Arnowitt and A. Chamseddine, Applied $N=1$ Supergravity, ICTP Series in Theoretical Physics, Vol.I, World Scientific (1984); R. Arnowitt and P. Nath, Lectures presented at VII J. A. Swieca Summer School, Campos do Jordao, Brazil, 1993, CTP-TAMU-52/93

[6] K. Inoue, A. Kakuto, H. Komatsu and H. Takeshita, Prog. Theor. Phys. 68, 927 (1982) and $\mathbf{7 1}, 413$ (1984).

[7] Some of the many recent papers include, along with Ref. [8], J. Ellis and F. Zwirner, Nucl. Phys. B338, 317 (1990); G. Ross and R. G. Roberts, Nucl. Phys. B377, 571 (1992); R. Arnowitt and P. Nath, Phys. Rev. Lett. 69, 725 (1992); S. Kelley, J. Lopez, D. Nanopoulos, H. Pois and K. Yuan, Nucl. Phys. B398, 3 (1993); G. Kane, C. Kolda, L. Roszkowski and J. Wells, UM-TH-93-24 (1993); V. Barger, M. Berger and P. Ohmann, $\mathrm{MAD} / \mathrm{PH} / 801$ (1994). References to earlier literature may be found in these.

[8] M. Drees and M. Nojiri, Nucl. Phys. B369, 54 (1992).

[9] S. Martin and M. Vaughn, North-Eastern preprint, NUB-3072-93 (1993)

[10] B. Campbell, Phys. Rev. D28, 209 (1983).

[11] H. Arason et. al. Phys. Rev. Lett. 67, 2933 (1991) and Phys. Rev. D47, 232 (1993).

[12] V. Barger, M. S. Berger, P. Ohmann, Phys. Rev. D47, 1093 (1993); V. Barger, M. S. Berger, P. Ohmann and R. J. N. Phillips, Phys. Lett. 314B, 351 (1993).

[13] M. Carena, S. Pokorski and C. Wagner, Nucl. Phys. B406, 59 (1993).

[14] P. Langacker and N. Polonsky, Pennsylvania preprint, UPR-0556-T (1993).

[15] See e.g. The Solenoidal Detector Collaboration Technical Design Report; GEM Collaboration, Technical Design Report; Report of the Supersymmetry Working Group in Proceedings of the Large Hadron Collider Workshop, Aachen, October 1990, CERN9010; F. Pauss, ibid; H. Baer et. al. in Research Directions for the Decade, E. Berger, Editor (World Scientific, 1992).

[16] H. Baer, X. Tata and J. Woodside, Phys. Rev. D45, 142 (1992).

[17] J. Ellis, K. Enqvist, D. Nanopoulos and K. Tamvakis, Phys. Lett. 155B, 381 (1985); M. Drees, ibid. 158B, 409 (1985) and Phys. Rev. D33, 1468 (1986).

[18] F. Paige and S. Protopopescu, in Supercollider Physics, p. 41, ed. D. Soper (World Scientific, 1986); H. Baer, F. Paige, S. Protopopescu and X. Tata, in the Proceedings of the Workshop on Physics at Current Accelerators and the Supercollider, ed. J. Hewett, A. White and D. Zeppenfeld, Argonne National Laboratory Report ANL-HEP-CP-93-92 (1993).

[19] We do not consider the large $\tan \beta$ solution corresponding to the fixed point of the 
bottom Yukawa interaction because radiative breaking constraints require that $\tan \beta \leq$ $m_{t} / m_{b}$.

[20] See e.g. P. Langacker, Invited Talk at 30 Years of Neutral Currents. From Weak Neutral Currents to W/Z and Beyond, Santa Monica, February 1993.

[21] P. Nath, A. Chamseddine and R. Arnowitt, Phys. Rev. D32, 3248 (1985); J. Hisano, H. Murayama and T. Yanagida, Nucl. Phys. B402, 46 (1993).

[22] R. Arnowitt and P. Nath, Phys. Rev. Lett. 69, 725 (1992), which takes into account the constraints from radiative symmetry breaking, have concluded that the non-observation of proton decay implies the bound $m_{0} \geq 500 \mathrm{GeV}$; while we have not incorporated their more restrictive analysis into our considerations, we mention that this bound is respected by four of the six cases that we study in the following.

[23] K. Griest and D. Seckel, Phys. Rev. D43, 3191 (1991); P. Gondolo and G. Gelmini, Nucl. Phys. B360, 145 (1991); P. Nath and R. Arnowitt, Phys. Rev. Lett. 70, 3696 (1993); M. Drees and M. Nojiri, Phys. Rev. D47, 376 (1993).

[24] Y. Okada, M. Yamaguchi and T. Yanagida, Phys. Lett. 262B, 54 (1991) and Prog. Theor. Phys. 85, 1 (1991); J. Ellis, G. Ridolfi and F. Zwirner, Phys. Lett. 257B, 83 (1991).

[25] The arguments for not considering the mass ratios for the $T_{3}=-1 / 2$ fermions for the first two generations are the same as for standard $\mathrm{SU}(5)$. We also remind the reader that we have allowed for some variation of $m_{b} / m_{\tau}$ to allow for threshold effects and effects of suppressed non-renormalizable operators.

[26] H. Baer, M. Bisset, X .Tata and J. Woodside, Phys. Rev. D46, 303 (1992).

[27] The incorporation of the pole gluino mass can substantially change the phenomenology especially when the gluino is very light.

[28] H. Baer, M. Drees, R. Godbole, J. Gunion and X. Tata, Phys. Rev. D44, 725 (1991).

[29] H. Baer, J. Sender and X. Tata, (in preparation).

[30] K. Hikasa and M. Kobayashi, Phys. Rev. D36, 724 (1987).

[31] H. Baer, C. Kao and X. Tata, Phys. Rev. D48, 5175 (1994).

[32] H. Baer, M. Bisset, X. Tata and J. Woodside, Phys. Rev. D46, 303 (1992).

[33] G. Gopal, presented at the Aspen Winter Conference on Particle Physics Beyond the Year 2000, Aspen, CO, January, 1994.

[34] See e.g. H. Eberl and W. Majerotto, Vienna Preprint, HEPHT-PUB 595/93 (1993).

[35] E. Eichten, I. Hinchliffe, K. Lane and C. Quigg, Rev. Mod. Phys. 56, 759 (1984).

[36] H. Baer, V. Barger, D. Karatas and X. Tata, Phys. Rev. D36, 96 (1987).

[37] R. M. Barnett, J. F. Gunion and H. E. Haber, Phys. Lett. 315, 349 (1993).

[38] D. Dicus and R. Vega, Phys. Lett. B217, 194 (1989).

[39] H. Baer, C.H. Chen, F. Paige and X. Tata, FSU-HEP-940310 (1994).

[40] J. Gunion and H. Pois, UCD-94-1 (1994). 


\section{TABLES}

TABLE I. Parameters and masses for six SUGRA cases A1, A2 and B1-B4.

\begin{tabular}{lrrrrrr}
\hline \hline parameter & $\mathrm{A} 1$ & $\mathrm{~A} 2$ & $\mathrm{~B} 1$ & $\mathrm{~B} 2$ & $\mathrm{~B} 3$ & $\mathrm{~B} 4$ \\
\hline$m_{0}$ & 500 & 500 & 300 & 1000 & 400 & 1000 \\
$m_{\frac{1}{2}}$ & 120 & 130 & 60 & 70 & 130 & 130 \\
$A_{0} / m_{0}$ & 0.6 & 3.75 & 0.1 & 0.0 & 0.0 & 0.0 \\
$\tan \beta$ & 1.49 & 2.2 & 1.94 & 1.32 & 2.22 & 2.11 \\
$\mu$ & 697.1 & 580 & -313.3 & -1571.7 & 430 & 964.7 \\
$m_{t}$ & 160 & 175 & 170 & 155 & 175 & 175 \\
$m_{\tilde{g}}$ & 346 & 371 & 185 & 231 & 364 & 400 \\
$m_{\tilde{q}}$ & 568 & 578 & 328 & 1011 & 496 & 1039 \\
$m_{\tilde{t}_{1}}$ & 131 & 83.6 & 198 & 121 & 225 & 288 \\
$m_{\tilde{t}_{2}}$ & 521 & 500 & 304 & 781 & 470 & 799 \\
$m_{\tilde{b}_{L}}$ & 437 & 426 & 250 & 732 & 396 & 765 \\
$m_{\tilde{\ell}}$ & 508 & 501 & 305 & 1001 & 412 & 1005 \\
$m_{\widetilde{Z}_{1}}$ & 43.6 & 48.0 & 27.1 & 28.8 & 46.1 & 50.7 \\
$m_{\widetilde{Z}_{2}}$ & 85.1 & 93.5 & 63.5 & 59.8 & 89.3 & 99.9 \\
$m_{\widetilde{Z}_{3}}$ & 698 & 582 & 316 & 1572 & 432 & 966 \\
$m_{\widetilde{Z}_{4}}$ & 710 & 595 & 333 & 1577 & 450 & 973 \\
$m_{\widetilde{W}_{1}}$ & 84.5 & 92.9 & 62.2 & 59.7 & 87.9 & 99.6 \\
$m_{\widetilde{W}_{2}}$ & 707 & 593 & 331 & 1576 & 448 & 972 \\
$m_{H_{\ell}}$ & 85.2 & 99.7 & 61.6 & 86.0 & 91.3 & 101 \\
$m_{H_{h}}$ & 1038 & 841 & 492 & 2339 & 652 & 1543 \\
$m_{H_{p}}$ & 1038 & 842 & 487 & 2342 & 649 & 1542 \\
$m_{H}$ & 1040 & 845 & 492 & 2343 & 653 & 1544 \\
\hline \hline & & & & & & \\
\hline
\end{tabular}


TABLE II. (a) Fractions of SUSY particle pairs produced in $p p$ collisions at the LHC; and (b) branching fractions of selected decay modes, for six SUGRA cases A1, A2 and B1-B4, where $\tilde{q}$ stands for all squarks except stops, and $\tilde{\chi} \widetilde{\chi}$ stands for all possible chargino and neutralino pairs: $\widetilde{W}_{i} \widetilde{W}_{j}, \widetilde{Z}_{i} \widetilde{Z}_{j}$, and $\widetilde{W}_{i} \widetilde{Z}_{j}$.

\begin{tabular}{|c|c|c|c|c|c|c|}
\hline SUSY particles $\backslash$ Case & A1 & $\mathrm{A} 2$ & B1 & $\mathrm{B} 2$ & B3 & B4 \\
\hline \multicolumn{7}{|c|}{ (a) SUSY Particle Pairs Produced } \\
\hline$\tilde{g} \tilde{g}$ & 0.30 & 0.093 & 0.72 & 0.74 & 0.44 & 0.73 \\
\hline$\tilde{t} \tilde{t}$ & 0.51 & 0.84 & 0.011 & 0.21 & 0.10 & 0.083 \\
\hline$\tilde{g} \tilde{q}$ & 0.13 & 0.050 & 0.23 & 0.013 & 0.33 & 0.081 \\
\hline$\tilde{q} \tilde{q}$ & 0.018 & 0.007 & 0.029 & $3 \times 10^{-4}$ & 0.067 & 0.005 \\
\hline$\widetilde{W}_{1}^{ \pm} \widetilde{Z}_{2}$ & 0.018 & 0.006 & 0.004 & 0.019 & 0.027 & 0.066 \\
\hline$\tilde{\chi} \tilde{\chi}$ & 0.026 & 0.009 & 0.007 & 0.027 & 0.042 & 0.088 \\
\hline \multicolumn{7}{|c|}{ (b) Important Decay Modes } \\
\hline$\tilde{g} \rightarrow \tilde{t}_{1} \bar{t}, \tilde{t}_{1} t$ & 1.0 & 1.0 & - & - & - & - \\
\hline$\tilde{g} \rightarrow \widetilde{W}_{1}^{-} t \bar{b}, \widetilde{W}_{1}^{+} b \bar{t}$ & $1.6 \times 10^{-4}$ & $1.4 \times 10^{-4}$ & - & 0.091 & 0.12 & 0.25 \\
\hline$\tilde{g} \rightarrow \widetilde{W}_{1}^{-} u \bar{d}, \widetilde{W}_{1}^{+} d \bar{u}$ & $4.6 \times 10^{-4}$ & $2.9 \times 10^{-4}$ & 0.21 & 0.10 & 0.19 & 0.16 \\
\hline$\tilde{g} \rightarrow \widetilde{Z}_{1} d \bar{d}$ & $3.1 \times 10^{-5}$ & $1.9 \times 10^{-5}$ & 0.012 & 0.005 & 0.014 & 0.01 \\
\hline$\tilde{g} \rightarrow \widetilde{Z}_{1} b \bar{b}$ & $6.8 \times 10^{-5}$ & $5.4 \times 10^{-5}$ & 0.012 & 0.006 & 0.035 & 0.018 \\
\hline$\tilde{g} \rightarrow \widetilde{Z}_{2} b \bar{b}$ & $4.3 \times 10^{-4}$ & $3.9 \times 10^{-4}$ & 0.21 & 0.10 & 0.18 & 0.15 \\
\hline$\tilde{t}_{1} \rightarrow \widetilde{W}_{1}^{+} b$ & 1.0 & - & 0.95 & 1.0 & 0.93 & 0.29 \\
\hline$\tilde{t}_{1} \rightarrow \widetilde{Z}_{1} t$ & - & - & 0.05 & - & 0.07 & 0.64 \\
\hline$\tilde{t}_{1} \rightarrow \widetilde{Z}_{2} t$ & - & - & - & - & - & 0.07 \\
\hline$\tilde{t}_{1} \rightarrow \widetilde{Z}_{1} c$ & - & 1.0 & - & - & - & - \\
\hline$\widetilde{Z}_{2} \rightarrow \widetilde{Z}_{1} d \bar{d}$ & 0.11 & 0.18 & 0.024 & 0.028 & 0.21 & 0.17 \\
\hline$\widetilde{Z}_{2} \rightarrow \widetilde{Z}_{1} b \bar{b}$ & 0.37 & 0.39 & 0.057 & 0.22 & 0.38 & 0.42 \\
\hline$\widetilde{Z}_{2} \rightarrow \widetilde{Z}_{1} e^{+} e^{-}$ & 0.047 & 0.018 & 0.14 & 0.12 & 0.007 & 0.012 \\
\hline$\widetilde{W}_{1}^{+} \rightarrow \widetilde{Z}_{1} e^{+} \nu_{e}$ & 0.11 & $6.9 \times 10^{-5}$ & 0.11 & 0.11 & 0.11 & 0.11 \\
\hline
\end{tabular}


TABLE III. Cross sections in $p b$ for various event topologies after cuts described in the text, for $p p$ collisions at $\sqrt{s}=14 \mathrm{TeV}$. The various SUGRA cases are listed in the first column. The OS/SS ratio is computed with the OS dilepton sample before the scalar $E_{T}$ cut.

\begin{tabular}{cccccccc}
\hline \hline case & $E_{T}$ & $1 \ell$ & $O S$ & $S S$ & OS/SS & $3 \ell$ & $4 \ell$ \\
\hline A1 & 24.6 & 36.2 & 5.4 & 3.7 & 2.0 & 1.2 & 0.017 \\
A2 & 48.0 & 31.4 & 1.5 & 2.1 & 1.2 & $<0.02$ & $<0.02$ \\
B1 & 79.1 & 76.8 & 11.9 & 3.4 & 6.9 & 1.7 & 0.17 \\
B2 & 67.7 & 37.5 & 9.0 & 2.4 & 7.7 & 0.8 & 0.1 \\
B3 & 51.8 & 21.2 & 1.4 & 0.6 & 3.3 & 0.09 & $<0.01$ \\
B4 & 20.1 & 10.1 & 1.1 & 0.4 & 3.5 & 0.1 & $<0.004$ \\
BTW1 & 105 & 39.8 & 2.8 & 1.4 & 3.1 & 0.21 & 0.03 \\
BTW2 & 57.3 & 22.5 & 2.2 & 0.85 & 3.6 & 0.14 & $<0.02$ \\
BTW3 & 96 & 58 & 10.9 & 2.9 & 6.7 & 1.5 & 0.06 \\
BTW4 & 52.3 & 23.9 & 2.3 & 0.9 & 3.7 & 0.2 & $<0.02$ \\
t $\bar{t}(160)$ & 2.9 & 8.0 & 1.1 & 0.01 & 640 & $<0.004$ & $<0.004$ \\
$W+j e t$ & 0.6 & 3.8 & 0.29 & - & & - & - \\
$W W$ & - & - & 0.001 & - & & - & - \\
$Z+j e t$ & 0.6 & 0.2 & 0.02 & - & & - & - \\
$b \bar{b}, c \bar{c}$ & 0.2 & 0.02 & - & - & & & - \\
total BG & 4.3 & 12.02 & 1.411 & 0.01 & & & - \\
\hline \hline
\end{tabular}

TABLE IV. Cross sections in pb for various event topologies (before) after the cuts on scalar $E_{T}$ and $M_{T}$ described in Sec. $\mathrm{V}$ of the text, for $p p$ collisions at $\sqrt{s}=14 \mathrm{TeV}$. The various SUGRA cases are listed in the first column.

\begin{tabular}{cccc}
\hline \hline case & $\not_{T}$ & $1 \ell$ & $O S$ \\
\hline A1 & $(26.3) 24.6$ & $(62.6) 36.2$ & $(7.3) 5.4$ \\
A2 & $(53.9) 48.0$ & $(50.1) 31.4$ & $(2.5) 1.5$ \\
B1 & $(103) 79.1$ & $(209) 76.8$ & $(23.5) 11.9$ \\
B2 & $(93.0) 67.7$ & $(122) 37.5$ & $(18.6) 9.0$ \\
B3 & $(56.8) 51.8$ & $(35.0) 21.2$ & $(2.0) 1.4$ \\
B4 & $(21.7) 20.1$ & $(16.3) 10.1$ & $(1.4) 1.1$ \\
BTW1 & $(127) 105$ & $(85.5) 39.8$ & $(4.5) 2.8$ \\
BTW2 & $(65.4) 57.3$ & $(42.0) 22.6$ & $(3.1) 2.2$ \\
BTW3 & $(119) 96.0$ & $(127) 58.1$ & $(19.3) 10.9$ \\
BTW4 & $(58.9) 52.3$ & $(52.4) 8.0$ & $(3.2) 2.3$ \\
$t \bar{t}(160)$ & $(4.6) 2.9$ & $(108) 3.8$ & $(6.4) 1.1$ \\
$W+j e t$ & $(1.7) 0.6$ & $(1.4) 0.2$ & $(3.8) 0.29$ \\
$Z+j e t$ & $(1.2) 0.6$ & $(0.13) 0.02$ & $(0.10) 0.02$ \\
$b \bar{b}, c \bar{c}$ & $(0.24) 0.20$ & $(161.9) 12.02$ & $-(-)$ \\
total BG & $(7.7) 4.3$ & & $10.3(1.41)$ \\
\hline \hline
\end{tabular}


TABLE V. Cross sections and event fractions for missing energy plus jets events for various SUSY cases at the LHC.

\begin{tabular}{ccccccccc}
\hline \hline case & $\sigma(p b)$ & $n_{j}=4-5$ & $n_{j}=6-7$ & $n_{j} \geq 8$ & $n_{b} \geq 1$ & $n_{b} \geq 2$ & $\left\langle\Sigma E_{T}\right\rangle$ & $\left\langle E_{T}>\right.$ \\
\hline A1 & 24.6 & 0.54 & 0.35 & 0.10 & 0.65 & 0.28 & 1160 & 212 \\
A2 & 48.0 & 0.55 & 0.35 & 0.10 & 0.47 & 0.09 & 1112 & 221 \\
B1 & 79.1 & 0.73 & 0.25 & 0.02 & 0.21 & 0.04 & 964 & 195 \\
B2 & 67.7 & 0.77 & 0.20 & 0.03 & 0.23 & 0.05 & 999 & 211 \\
B3 & 51.8 & 0.57 & 0.35 & 0.08 & 0.36 & 0.12 & 1118 & 215 \\
B4 & 20.1 & 0.54 & 0.36 & 0.10 & 0.44 & 0.15 & 1204 & 217 \\
BTW1 & 105 & 0.69 & 0.26 & 0.04 & 0.17 & 0.04 & 1006 & 214 \\
BTW2 & 57.3 & 0.61 & 0.33 & 0.05 & 0.18 & 0.04 & 1091 & 211 \\
BTW3 & 96 & 0.69 & 0.27 & 0.04 & 0.15 & 0.03 & 1011 & 217 \\
BTW4 & 52.3 & 0.60 & 0.33 & 0.06 & 0.18 & 0.04 & 1109 & 208 \\
$t \bar{t}(160)$ & 2.9 & 0.81 & 0.17 & 0.01 & 0.56 & 0.12 & 895 & 201 \\
$Z+j e t s$ & 0.63 & 0.89 & 0.11 & 0.0 & 0.11 & 0.02 & 905 & 281 \\
\hline \hline
\end{tabular}

TABLE VI. Cross sections and event fractions for events containing a single lepton plus jets for various SUSY cases at the LHC.

\begin{tabular}{ccccccccc}
\hline \hline case & $\sigma(p b)$ & $n_{j}=0-3$ & $n_{j}=4-5$ & $n_{j} \geq 6$ & $n_{b} \geq 1$ & $n_{b} \geq 2$ & $\left\langle\Sigma E_{T}\right\rangle$ & $\left\langle E_{T}>\right.$ \\
\hline A1 & 36.2 & 0.23 & 0.49 & 0.28 & 0.67 & 0.29 & 1056 & 167 \\
A2 & 31.4 & 0.26 & 0.54 & 0.21 & 0.45 & 0.10 & 1020 & 191 \\
B1 & 76.8 & 0.35 & 0.50 & 0.15 & 0.27 & 0.06 & 913 & 148 \\
B2 & 37.5 & 0.41 & 0.50 & 0.09 & 0.23 & 0.04 & 910 & 165 \\
B3 & 21.2 & 0.24 & 0.53 & 0.24 & 0.36 & 0.1 & 1052 & 178 \\
B4 & 10.1 & 0.22 & 0.52 & 0.26 & 0.46 & 0.16 & 1108 & 185 \\
BTW1 & 39.8 & 0.32 & 0.52 & 0.15 & 0.15 & 0.03 & 970 & 176 \\
BTW2 & 22.6 & 0.26 & 0.54 & 0.19 & 0.15 & 0.03 & 1030 & 172 \\
BTW3 & 58 & 0.33 & 0.52 & 0.15 & 0.15 & 0.03 & 969 & 174 \\
BTW4 & 23.9 & 0.24 & 0.55 & 0.20 & 0.15 & 0.04 & 1024 & 168 \\
$t \bar{t}(160)$ & 8 & 0.49 & 0.46 & 0.04 & 0.52 & 0.12 & 857 & 153 \\
$W+j e t s$ & 3.8 & 0.92 & 0.08 & 0.0 & 0.08 & 0.0 & 927 & 208 \\
\hline \hline
\end{tabular}


TABLE VII. Cross sections and event fractions for events containing opposite sign dileptons plus jets for various SUSY cases at the LHC.

\begin{tabular}{cccccccccc}
\hline \hline case & $\sigma(p b)$ & $n_{j}=0-3$ & $n_{j}=4-5$ & $n_{j} \geq 6$ & $n_{b} \geq 1$ & $n_{b} \geq 2$ & $\left\langle\Sigma E_{T}\right\rangle$ & $\left\langle E_{T}\right\rangle$ & $A_{f}$ \\
\hline A1 & 5.4 & 0.33 & 0.47 & 0.20 & 0.69 & 0.32 & 1017 & 165 & $-0.009 \pm 0.06$ \\
A2 & 1.5 & 0.39 & 0.47 & 0.14 & 0.47 & 0.11 & 950 & 161 & $0.05 \pm 0.16$ \\
B1 & 11.9 & 0.54 & 0.41 & 0.06 & 0.35 & 0.14 & 895 & 135 & $0.77 \pm 0.07$ \\
B2 & 9.0 & 0.42 & 0.51 & 0.07 & 0.26 & 0.07 & 920 & 163 & $0.79 \pm 0.05$ \\
B3 & 1.4 & 0.29 & 0.50 & 0.21 & 0.53 & 0.15 & 1035 & 179 & $0.32 \pm 0.08$ \\
B4 & 1.1 & 0.30 & 0.54 & 0.16 & 0.58 & 0.25 & 1050 & 183 & $0.26 \pm 0.05$ \\
BTW1 & 2.8 & 0.41 & 0.50 & 0.08 & 0.20 & 0.03 & 942 & 166 & $0.7 \pm 0.07$ \\
BTW2 & 2.2 & 0.29 & 0.52 & 0.19 & 0.23 & 0.03 & 1042 & 162 & $0.6 \pm 0.07$ \\
BTW3 & 10.9 & 0.39 & 0.51 & 0.10 & 0.17 & 0.03 & 984 & 165 & $0.78 \pm 0.03$ \\
BTW4 & 2.3 & 0.36 & 0.50 & 0.14 & 0.21 & 0.03 & 990 & 156 & $0.77 \pm 0.05$ \\
$t \bar{t}(160)$ & 1.1 & 0.60 & 0.38 & 0.02 & 0.50 & 0.12 & 827 & 144 & $0.01 \pm 0.06$ \\
\hline \hline
\end{tabular}




\section{FIGURES}

FIG. 1. Transverse momentum distribution of fastest jet in multi-jet plus $\not_{T}$ events for four SUGRA cases and the sum of backgrounds (histogram), at $\sqrt{s}=14 \mathrm{TeV}$.

FIG. 2. Distributions for a) scalar $E_{T}, b$ ) lepton plus missing energy transverse mass, and c) $p_{T}$ (lepton) in single isolated lepton events for four SUGRA cases A1 (dashes), A2 (dot-dash), B1 (double-dot-dash) and B4 (triple-dot-dash), along with SM background (solid histogram), at $\sqrt{s}=14 \mathrm{TeV}$. Distributions $a$ ) and $b$ ) are made before scalar $E_{T}$ and $m_{T}$ cuts, while $c$ ) is after cuts.

FIG. 3. Distributions for $a$ ) scalar $E_{T}, b$ ) fast lepton transverse momentum, and $c$ ) slow lepton transverse momentum in opposite sign isolated dilepton events for four SUGRA cases A1 (dashes), A2 (dot-dash), B2 (double-dot-dash) and B4 (triple-dot-dash), along with SM background (solid histogram), at $\sqrt{s}=14 \mathrm{TeV}$. Distribution $a$ ) is made before the scalar $E_{T}$ cut, while $b$ ) and $c$ ) are after the scalar $E_{T}$ cut.

FIG. 4. Distributions for dilepton opening angle in the transverse plane for OS dileptons and SS dileptons for SUGRA cases A1, B2 and B4.

FIG. 5. Distributions for a) fast lepton transverse momentum, and b) slow lepton transverse momentum in same sign isolated dilepton events for four SUGRA cases A1 (dashes), A2 (dot-dash), B2 (double-dot-dash) and B4 (triple-dot-dash), along with SM background (solid histogram), at $\sqrt{s}=14 \mathrm{TeV}$. 
This figure "fig1-1.png" is available in "png" format from: http://arxiv.org/ps/hep-ph/9403307v1 
This figure "fig2-1.png" is available in "png" format from: http://arxiv.org/ps/hep-ph/9403307v1 
This figure "fig1-2.png" is available in "png" format from: http://arxiv.org/ps/hep-ph/9403307v1 
This figure "fig2-2.png" is available in "png" format from: http://arxiv.org/ps/hep-ph/9403307v1 
This figure "fig2-3.png" is available in "png" format from: http://arxiv.org/ps/hep-ph/9403307v1 